\title{
SOBRE EL ESTATUTO LINGÜÍSTICO DE LAS LENGUAS DE SEÑAS ${ }^{1}$
}

\author{
Mariano Reyes Tejedor
}

Universidad Pablo de Olavide

\begin{abstract}
RESUMEN
El objetivo de este articulo es reivindicar que las lenguas de señas o signos son sistemas lingüisticos naturales y no códigos artificiales de comunicación y que, por tanto, deben ser estudiadas como tales desde las disciplinas cientificas. Con este objetivo, presentamos evidencias Neurolingüisticas y Psicolingüisticas que contradicen este prejuicio lingüistico arraigado en nuestra sociedad e incluso en ámbitos académicos universitarios.

Palabras clave: lenguas de signos o señas, prejuicios lingüísticos, neurolingüística, psicolingüística, clasificadores.

ABSTRACT

The aim of this article is to vindicate that signed languages are natural linguistic systems and not artificial communication codes. Therefore, as such they should be considered by researchers. With this goal in mind, Neurolinguistics and Psycholinguistic evidences, which challenge this linguistic prejudice deep-rooted in our society and even in academic environments, are presented.
\end{abstract}

Keywords: signed languages, linguistic prejudices, neurolinguistics, psycholinguistics, classifiers.

\section{INTRODUCCIÓN}

¿Por qué la sociedad considera que las lenguas de señas son lenguas inferiores o secundarias en relación con las lenguas orales? Parece ser el reflejo de un prejuicio lingǘstico decimonónico arraigado en nuestra sociedad que hace que pensemos erróneamente que existen lenguas de primera, segunda y tercera división. En la categoría de las lenguas de primera división se incluirían las lenguas orales que han sido vehículo del desarrollo cultural de la humanidad, también entre ellas habría cierta jerarquía; la categoría de segunda división contendría el resto de lenguas orales habladas por los integrantes de culturas indígenas de pueblos no desarrollados que, no tienen o no han

\footnotetext{
${ }^{1}$ Quiero agradecer las sugerencias que me han aportado durante la realización de este artículo a Juan Carlos Moreno Cabrera, José Gabriel Storch de Gracia y Victoria Camacho Taboada. Por supuesto, ellos no son responsables de mis opiniones o de los errores que haya podido cometer.
} 
tenido hasta hace poco escritura y, por último, se encuentran las lenguas de tercera división, las lenguas de señas que ni siquiera son consideradas sistemas lingüísticos naturales. Sin embargo, la lingüística actual nos apunta que todas estas lenguas tienen el mismo estatuto de lenguas naturales, aunque, por supuesto, cada una de ellas tenga sus propias particularidades. Una postura que contrasta con los siguientes comentarios de Leonard Bloomfield:

Some communities have a gesture language which upon occasion they use instead of speech. (...) It seems that these gesture languages are merely developments of ordinary gestures and that any and all complicated or not immediately intelligible gestures are based on the conventions of ordinary speech (Bloomflied, 1933:39).

Otra de las causas puede deberse al hecho de que la mayoría de nuestros congéneres usa como medio de comunicación lenguas orales. Como consecuencia, suele decirse que la evolución ha favorecido el habla como modalidad lingüística y que, por tanto, los bebés cuando nacen estarían predispuestos a desarrollar exclusivamente una lengua oral y no una lengua de señas.

En este artículo intentaremos demostrar que las lenguas de señas son lenguas naturales con el mismo estatuto lingüístico que las lenguas orales, a excepción de que usan la modalidad gestual-visual, y no la modalidad auditiva-oral (véase Stokoe, 1960; Klima y Bellugi, 1979; Sandler y Lillo-Martin, 2001; Emmorey, 2002 o Sandler y LilloMartin, 2006). Para ello comenzaremos describiendo algunos intentos de enseñar la lengua de señas a primates, y el fracaso que esto supuso. Una prueba de que estas lenguas tienen la misma complejidad formal que las orales y que no se trata de simples códigos. Es necesario señalar, no obstante, que dichos experimentos no estaban diseñados para demostrar que las lenguas de signos tuvieran el estatuto lingüístico que las orales. Seguidamente, nos centraremos en estudios recientes neurolingǘsticos que parecen demostrar que, ciertamente, la Facultad Humana del Lenguaje usa indistintamente la modalidad visual-gestual y la oral-auditiva y que ambas, la lengua oral y la no oral, se procesan en el mismo hemisferio cerebral, el izquierdo. Finalmente, presentaremos algunas evidencias psicolingüísticas en las que se comprueba que las lenguas de señas se adquieren y desarrollan de forma natural.

\section{LA FACUlTAD HuMANA DEL LENGUAJE}

Parece demostrado que la facultad lingüística humana es una capacidad exclusiva de nuestra especie. El lenguaje humano se caracteriza por ser un sistema de signos estructurado capaz de generar un conjunto ilimitado, en teoría, infinito de expresiones lingüísticas. Esta creatividad característica es consecuencia de la propiedad de la infinitud discreta o uso infinito de medios finitos, propiedad ya observada por Descartes y Galileo que ha sido resaltada en el siglo XX por Noam Chomsky (1965).

Durante los años setenta del pasado siglo, diversos experimentos fueron llevados a cabo en EEUU mayoritariamente por psicólogos con el objetivo de comprobar hasta 
qué punto chimpancés y gorilas podían adquirir el lenguaje humano. Aunque los primeros intentos se hicieron con lengua oral, debido a las características neuroanatómicas de estos animales se decidió usar como lengua de referencia la Lengua de Señas Americana (ASL). ${ }^{2}$

Los experimentos se llevaron a cabo haciendo uso del método conductista. Los chimpancés aprendían las señas mediante la práctica intensiva y la esperanza de obtener una recompensa inmediata. Tras varios años de entrenamiento estos animales consiguieron adquirir un número significativo de señas que aparentemente servían para la comunicación (Gardner y Gardner, 1969; Terrace, et al. 1979).

Así, los Gardner tras tres años enseñando la lengua de señas a la chimpancé Washoe, aseguraron que ésta fue capaz de aprender unos 132 signos gestuales similares, en cierto sentido, a las señas de la ASL (véase nota al pie 2) (Gardner y Gardner, 1969). Washoe no creció en un entorno humano, sino que vivió en un contexto de semilibertad. En su presencia estaba prohibido hablar inglés para evitar que influyera en la comunicación. Los Gardner aseguraban que Washoe se comunica intencionalmente con signos arbitrarios de manera creativa. Relatan, por ejemplo, que conocía las señas correspondientes a AGUA y PÁJARO y que en cierta ocasión ante la presencia de un pato señó el compuesto AGUA+PÁJARO.

En principio, esto pudiera ser interpretado como un ejemplo de creatividad léxica. Sin embargo, es posible que Washoe estuviera simplemente haciendo referencia sucesivamente al agua y al pájaro y que no se tratara de una forma compuesta tal y como defendían sus cuidadores. Los resultados del experimento Washoe son controvertidos, además se carece de las grabaciones que pudieran servir de prueba para confirmar o negar la creatividad lingüística que según sus cuidadores mostraba el primate.

Otro de los experimentos en el que se trató de demostrar la potencialidad de los primates en adquirir una lengua estructurada partió de la Universidad de Columbia (EEUU). Dicho experimento fue dirigido por Herbert Terrace y contaría con la participación. El experimento fue grabado enteramente en vídeo (Terrace et al., 1979).

El chimpancés objeto de estudio recibió el nombre de Nim Chimsky en honor al famoso lingüista Noam Chosmky. El método de trabajo fue básicamente el mismo que se usó con Washoe. Tras cuatro años de entrenamiento consiguieron que Nim aprendiera unas 125 señas que aparentemente combinaba para formar mensajes más complejos.

Tras la revisión de las grabaciones de Nim, Terrace llegó a las siguientes conclusiones: Nim no parecía estar usando las señas tal y como lo hacemos los

${ }^{2}$ No obstante, debemos aclarar que en realidad en los experimentos no se usaba la LSA, sólo un subconjunto de señas y algunas estructuras simples concadenadas. Los encargados de tratar con los primates no eran usuarios de la LSA y las señas reproducidas por los primates pueden considerarse burdas imitaciones de las señas de esta lengua. 
humanos. En primer lugar, nunca iniciaba la interacción, no respetaba los turnos de la conversación y no era capaz de mantener ninguna conversación coherente. Sólo el $12 \%$ de las señas eran espontáneos, mientras el $40 \%$ eran repeticiones de los que acababa de hacer el entrenador. Los colaboradores del experimento al recoger los datos eliminaban las repeticiones de tal manera que lo que originalmente había sido una lista de repeticiones sin estructura, por ejemplo, COMER, COMER, COMER, MANZANA, MANZANA, MANZANA, COMER, MANZANA eran recogidas como COMER MANZANA dando la apariencia de una estructura concreta.

Ante estos resultados se llegó a la conclusión de que lenguaje humano y el lenguaje de los chimpancés son esencialmente diferente por tres razones fundamentales: En primer lugar, los chimpancés no tiene recursos suficientes para poder generar un conjunto ilimitado de expresiones, su vocabulario es poco numeroso y carece de reglas de combinación sistemáticas que le ayuden a enunciar quién bizo qué a quién. Las lenguas humanas, por el contrario, se caracterizan no solo por poder encadenar más de dos palabras sino también por poder generar un conjunto infinito de estructuras. Para ello, nuestra especie cuenta con un conjunto finito de unidades y un conjunto finito de reglas de combinación que nos permite construir ilimitadas expresiones estructuradas. Cada signo está relacionado con una categoría lingüística determinada (1b), estos signos lingüísticos o categorías son agrupados en sintagmas (1c) de manera que reciben una función sintáctica específica (1d) y, por último, estos sintagmas están conectados entre sí mediante relaciones de dependencia (1e): ${ }^{3}$

(1)

\begin{tabular}{|l|l|l|l|l|l|}
\hline a. & El & chimpancé & comió & las & castañas \\
\hline b. & Determinante & Sustantivo & verbo & Determinante & Sustantivo \\
\hline c. & Sintagma Nominal & Sintagma Verbal & \\
\hline d & Función Sujeto & Función Predicado & \\
\hline e. & Concordancia sujeto y verbo \\
\hline
\end{tabular}

En segundo lugar, las señas que usan los chimpancés varían de forma y carecen de estructura interna. Esto es, la seña para manzana no es exactamente la misma de una emisión a otra, sino que puede variar considerablemente sin que pueda ser predicha por ningún patrón. Los signos lingüísticos, aunque pueden variar, siempre se forman siguiendo ciertos parámetros de los que no se desvía el señante.

En tercer lugar, los signos gestuales que usan estos primates, aunque pueden ser de tipo arbitrario, están fuertemente contextualizados. Los primates hacen referencia a objetos concretos que están presentes en el momento de la comunicación. Por ejemplo, son capaces de relacionar un signo gestual con una referencia concreta como puede ser manzana, pero no logran adquirir el concepto de fruta puesto que es un signo

${ }^{3} \mathrm{El}$ análisis de (1) no pretende ser exhaustivo, solo ilustrar las diferentes relaciones y categorías que subyacen a las expresiones lingüísticas. 
lingǘstico que conlleva el concepto de clase. Son incapaces de relacionar este signo solo y exclusivamente con todos los elementos incluidos en este concepto, esto es; manzana, pera, plátano, kaki, etc. En otras palabras, la comunicación está ligada al estímulo y a su vez existe una referencia directa entre signo y significado. En cambio, el signo lingüístico se caracteriza por hacer referencia a conceptos concretos u abstractos que en ningún momento tienen que estar presentes en la comunicación.

Frente a lo visto arriba, se puede comprobar que las lenguas de señas tienen recursos suficientes para poder generar un conjunto ilimitado de expresiones, presentan un vocabulario amplio, y hacen uso de reglas de combinación sistemáticas que les permiten asignar papeles temáticos (quién bizo qué a quién), es decir, presentan un conjunto finito de unidades y un conjunto finito de reglas de combinación que nos permite construir ilimitadas expresiones estructuradas.

Además, los signos lingüísticos en la lengua de señas, aunque pueden variar, siempre se forman siguiendo ciertos parámetros de los que el señante no se desvía; y finalmente, el signo lingüístico hace referencias a conceptos u objetos concretos o abstractos, ausentes en el momento de la comunicación en la lengua de señas, al igual que ocurre en la lengua oral.

En definitiva, es imposible que un primate pueda adquirir una lengua ya sea oral o de señas ya que son el producto de una facultad humana específica de nuestra especie: el lenguaje. Aunque estudios recientes llevados a cabo por Savage-Rumbaugh confirmen que los bonobos realizan tareas de comprensión similares a un niño de 2:6 de edad (lo que conlleva la comprensión de estructuras de cierta complejidad y uso de un lenguaje simbólico espontáneo), su lenguaje carece de las características sintácticas básicas expuestas en (1) (Savage-Rumbaugh, 2000). Para tener éxito en la comunicación necesitan combinar palabras con gestos y hacer referencias continuas al contexto de la emisión.

De hecho, estudios comparativos realizados con otras especies parecen mostrar que, si bien compartimos distintas propiedades computacionales y perceptivas, la propiedad de la discreta infinitud no se observa en ninguna otra especie animal (Hauser, Chomsky y Fitch, 2002). Por tanto, el sistema gestual de comunicación que utilizan los primates no humanos en cautividad no puede ser considerada una lengua de signos. Las lenguas de signos son sistemas lingüísticos complejos cuyas propiedades formales no pueden ser adquiridas por esta especie.

\section{EVIDENCIAS NEUROLINGÜÍSTICAS}

El posible origen genético del lenguaje es una de las cuestiones más controvertidas que han sido discutidas en Lingüística a partir de la segunda mitad del siglo XX. En aquel entonces, el paradigma lingǘstico que dominaba las universidades estadounidenses era el estructuralista-conductista que negaba al lenguaje cualquier vinculación genética. El lenguaje es una conducta aprendida. Sin embargo, el aspecto creador del lenguaje humano y la propiedad de la infinitud discreta no puede ser 
explicado si asumimos que el proceso de adquisición del lenguaje consiste en un procedimiento de condicionamiento operativo.

Hoy por hoy, el debate se centra en delimitar qué aspectos del lenguaje están regulados por la genética y cuáles no. Evidentemente, las propiedades del lenguaje deben estar predeterminadas en nuestros genes, pero ¿están especificadas sus características lingüísticas, o solo sus propiedades generales que están presentes en otras capacidades cognitivas? La única manera de comprobar cuál de estas hipótesis es la adecuada sería demostrar que existen trastornos del lenguaje asociados a algún gen. Y esto es lo que ha ocurrido recientemente. En el año 2001, investigadores de la Universidad de Oxford publicaron en la revista Nature los resultados de sus investigaciones en las que demuestran que la enfermedad conocida como Trastorno Específico del Lenguaje (TEL) tiene un origen genético (Lai et al., 2001). El TEL lo sufren personas que se caracterizan por tener una dificultad importante de expresarse en su lengua materna. Su producción lingüística parece ser similar a la de una persona que ha aprendido una segunda lengua siendo adulto. Tienen dificultad para flexionar verbos, realizar concordancias, etc. quedando preservadas otras capacidades cognitivas. Incluso algunos sujetos muestran un cociente de inteligencia no verbal superior a la media. Desde hace unos años se sospechaba que podría tener un origen genético puesto que afectaba a varios miembros de una misma familia y su patrón de distribución era claramente genético. Parece ser que los individuos afectados por esta dolencia tienen una mutación en un gen al que han denominado FOXP2. Esto, por supuesto, no significa que este gen sea el responsable del lenguaje, pero sí que, al menos, ha sido localizado un gen de las capacidades necesarias para la actividad lingüística.

Evidentemente, si partimos de la idea de que las propiedades formales del lenguaje están especificadas de alguna manera en nuestros genes (aunque no haya un acuerdo unánime en este punto) sería razonable pensar que dicha capacidad debe estar controlada y regulada por un órgano del cuerpo. El órgano responsable de este comportamiento es el cerebro, concretamente, las áreas especializadas responsables del lenguaje. Las lenguas de señas, como las orales, es el producto de dicha especialización cerebral.

Desde hace más de un siglo, los neurólogos Paul Broca (1861) y Karl Wernicke (1874) demostraron que la facultad humana del lenguaje está controlada por ciertas áreas funcionales especializadas del cerebro. Estos neurólogos observaron que algunos de sus pacientes tras haber sufrido un trauma cerebral sufrían una alteración acusada de la conducta lingüística. Broca advirtió que uno de sus pacientes con una lesión frontal izquierda (tercera circunvolución del lóbulo frontal) tenía problemas para expresarse de manera adecuada, mientras que Wernicke observó que una lesión en el lóbulo temporal posterior izquierdo provocaba una incapacidad manifiesta para comprender la lengua. Ambas afasias recibirían el nombre de afasia de Broca y 
Wernicke respectivamente 4 . Aunque hoy en día los neurólogos coinciden en decir que la distinción entre ambas patologías es de índole funcional y no tanto específica de estas zonas, todos coinciden en señalar que el lenguaje está controlado fundamentalmente por el hemisferio izquierdo del córtex cerebral y que es fácil establecer una relación entre áreas cerebrales y patologías lingüísticas. Por el contrario, el hemisferio derecho estaría al tanto del control visual-espacial. Esta organización cerebral, sin duda, debe tener un origen genético.

Por la cercanía del área de Broca al córtex motor y de Wernicke al córtex auditivo, se creía que afasia de Broca y de Wernicke eran productos de dicha proximidad respectivamente, alterándose la articulación del habla en el primer caso, y la percepción y procesamiento del sonido en el segundo. ${ }^{5}$ Si las lenguas de señas son sistemas de comunicación distintos al lenguaje humano oral, las áreas cerebrales responsables de la comunicación en signantes deberían encontrarse en el hemisferio derecho (hemisferio encargado del control visual-espacial); concretamente: la producción, alrededor del área responsable de las actividades motóricas, y la compresión alrededor del área responsable de la interpretación visual. Pero, en contra de esta intuición, diferentes estudios realizados con afásicos signantes, realizados durante los años ochenta, demostraron que el hemisferio que controla el uso de la lengua de señas es el izquierdo (Hickok, Bellugi y Klima, 2002).

Experimentos recientes en el que han sido utilizadas técnicas de medición más modernas tales como la neuroimagen y métodos fMRI parecen confirmar esta hipótesis (Petitto et al. 2000; Hickok, Bellugi y Klima, 2002; Hickok, Love-Geffen y Klima, 2002).

Interesantes fueron los estudios neurolingüísticos llevados a cabo por Petitto et al. (2000), en los que se usó el PET (Tomografía de Emisión de Positrones). Los sujetos participantes en este experimento fueron once sordos profundos señantes de la Lengua de Señas Americana o la Lengua de Señas de Québec y diez oyentes usuarios de lenguas orales. Las conclusiones obtenidas parecen demostrar que: (1) Los sujetos

4 En la década de los sesenta del siglo XX Geschwind (1965) retomará dicho modelo localizacionista o conexionista. Incluye tres afasias más: la transcortical sensorial, la transcortical motora, y la anomia (esta última de difícil clasificación) (Caplan, 1988).

${ }^{5}$ Esta descripción es la asumida por el modelo neurofuncional del lenguaje. Grodzinsky y Finkel (1998) y Grodzinsky (2000) argumentan, en cambio, que esta vinculación directa entre tareas (comprensión y producción) y áreas cerebrales no está del todo clara. De hecho, numerosos estudios han demostrado que el área de Broca está involucrada en el proceso de comprensión de ciertas estructuras sintácticas. Concretamente, Grodzinsky propone que las estructuras afectadas son aquellas en las que se ha producido un desplazamiento de un constituyente sintagmático. Para una descripción más detallada de esta hipótesis véase Reyes y Camacho (2007). Por otra parte, autores como Philip Lieberman aseguran que en el procesamiento de la sintaxis participan tanto áreas corticales como subcorticales (Lieberman, 2007).

I.S.S.N. $1132-0265$

Philologia Hispalensis 21 (2007) 1-17 
señantes procesan piezas muy específicas de su lengua (equivalentes a palabras y partes de palabras) en las mismas áreas que los sujetos hablantes. Y (2) estas áreas coinciden con las áreas cerebrales tradicionalmente asociadas con el procesamiento del sonido. ¿Significa esto que las personas sordas usan las áreas asociadas al sonido de las lenguas orales para la lengua de señas? No, más bien esto implicaría que estas áreas no están asociadas al sonido, sino al lenguaje, a la capacidad lingüistica en general ya sea oral o gestual. Por tanto, son áreas que procesan tanto la información sonora como la visual.

Con todo, las imágenes provenientes de los afásicos signantes indican la existencia de ciertas diferencias entre la actividad cerebral implicada entre hablantes y señantes. Cuando estos últimos usan su lengua, se percibe un incremento de la afluencia de sangre en las áreas visuales primarias del lóbulo occipital. En este caso, se demuestra cómo la plasticidad cerebral permite que áreas especializadas para la visión sean usadas también para funciones lingüísticas.

En resumen, los investigadores concluyen que, si bien las áreas implicadas en el lenguaje de ambas modalidades parecen ser prácticamente las mismas, pueden observarse pequeñas diferencias debido al hecho de que utilizan distintas modalidades sensoriales. Esto parece demostrar que el diseño del cerebro esta preestablecido genéticamente para que desarrollemos una lengua existiendo suficiente plasticidad para adaptarse a ambas modalidades por igual. ${ }^{6}$

Investigaciones actuales en Lingüística concluyen que el estudio de las lenguas de señas se está convirtiendo en una poderosa herramienta para el estudio de la arquitectura del lenguaje humano así como las implicaciones en la neurobiología. Su similitud con la lengua oral está proporcionando datos de primer orden para el estudio de las propiedades universales del lenguaje humano (véase Sandler y Lillo-Martin, 2006). De hecho, tras numerosos estudios se ha llegado a la conclusión de que tanto el lenguaje señado como el oral descansan en las mismas bases neuronales, ambos se procesarían en el área de Broca (Emmorey, 1996). En este sentido el área de Broca desempeña una función importante que explicamos a continuación.

Como hemos visto, si partimos del hecho de que el área de Broca está situada en la parte anterior de la corteza primaria encargada de los movimientos y de que es el área responsable de la producción del habla, se podría llegar a la falsa creencia que dicha

\footnotetext{
${ }^{6}$ Estos datos parecen rebatir algunas de las teorías del origen del lenguaje en nuestra especie que asumen que las propiedades combinatorias del lenguaje humano son la consecuencia de un cambio de función de las redes neuronales usadas para el control secuencial de los movimientos de los órganos articulatorios bucofaciales (Lieberman, 1984, 1991, 2006) o de los movimientos de la mano (Wilkins y J. Wakefield, 1995). Si las propiedades formales del lenguaje humano son básicamente independientes de la modalidad con la que se desarrolle la competencia del hablante, posiblemente el hito evolutivo responsable del surgimiento de la infinitud discreta en nuestra especie es independiente de estas funciones, aunque el sistema articulatorio-perceptual preferido fuera el oral-auditivo.
} 
área funciona así por su cercanía a la corteza primaria responsable de los movimientos oro-laríngeos. Por tanto, podríamos suponer que esta área sólo se activaría para el lenguaje oral, puesto que presupone movimientos de los órganos responsables del movimiento muscular para la ejecución del habla. Ya hemos señalado que tal implicación es falsa.

Al respecto, Corina et al. (1999) realizaron un experimento para averiguar si el área de Broca está implicada en la realización y ejecución manual de la ASL. El experimento consistía en estimular una zona del área de Broca mientras los señantes realizaban tareas de designación de objetos. Los resultados muestran que los señantes fallaban en tareas que podríamos llamar fonéticas: articulaban con movimientos inespecíficos, con una configuración de las señas poco precisas. Al contrario, errores de sustituciones fonológicas y semánticas se producían exclusivamente cuando se estimulaba el giro supramarginal izquierdo en el mismo señante. De los resultados de estos experimentos se desprende que el área 44 de Brodmann (BA 44), localizada en el Área de Broca, está implicada en la realización fonética del la ASL.

Otra forma de estudiar las implicaciones cerebrales de las lenguas de señas es el análisis de los trastornos lingüísticos en señantes. Se han documentado casos de señantes con una lesión en le lóbulo frontal que presentaban alteraciones en la producción, siendo el tipo de afasia no fluente, como es de esperar también en usuarios de la lengua oral. En esos casos se comprobó que el área afectada era la de Broca (BA 44 y BA 45) (Corina, 1998, Polizner et. al., 1987). Por su parte, Hickok et al. (1996) estudiaron el lenguaje de R. S., una señante que había sufrido un infarto en el hemisferio izquierdo afectando a las zonas del lenguaje. Se comprobó que R. S. comprendía bien pero su producción era muy deficiente. Lo más llamativo de este caso radica en que R. S. presentaba un déficit significativo en la coordinación de las dos manos. Se concluyó que dicho déficit era análogo al déficit fonético característico en la lengua oral: imprecisión articulatoria e incorrecta ejecución de los movimientos realizados por los órganos buco-fonatorios (lengua, laringe, los labios, etc.).

A modo de conclusión, estos investigadores advirtieron una analogía clara entre la afasia no-fluente de R. S. y la afasia en hablantes. Según estos datos, el área de Broca no es el área exclusiva de la lengua oral, sino que en ella residiría una facultad general que implica tanto a la lengua oral como a la lengua de señas, independientemente de la modalidad sensorial.

Por su parte, en 2003 Karen Emmorey y Melissa Herzig realizaron un experimento con señantes para estudiar de qué forma los sustantivos y la producción de los aspectos espaciales producidos mediante construcciones clasificadoras.

Brevemente, en ASL, al igual que en la Lengua de Señas Española (LSE), para expresar la posición de un objeto respecto a otro puede utilizarse bien preposiciones o clasificadores. Los clasificadores representan la relación espacial entre objetos según la configuración de la mano. Por ejemplo, para representar un objeto largo y fino (UN PINCEL) extenderíamos el dedo índice, y para señalar que se encuentra dentro de un 
recipiente de forma cilíndrica (UN VASO) se realizaría la configuración "C" de la LSE. El dedo índice extendido se colocaría dentro del signo cilíndrico que representa "vaso". De esta forma, el usuario de la ASL construye la oración el pincel está dentro del vaso mediante clasificadores.
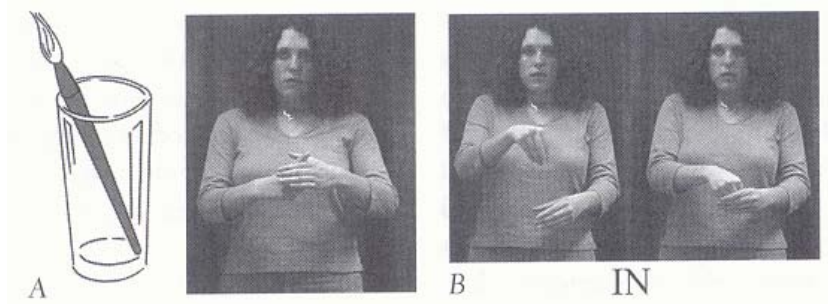

Fig. 1 Clasificador (A) y preposiciones EN (B) en ASL (Emmorey et al., 2006:174)

En este experimento se demostró una idea interesante: que la producción de dichos clasificadores no involucra al área de Broca, mientras que la producción de dichas relaciones a través de preposiciones del inglés oral sí lo hace. El experimento consistió en que bilingües en ASL y en lengua inglesa describieran la relación espacial existente entre dos objetos (sustantivos) en la primera lengua sin preposiciones y con clasificadores, y en la segunda con preposiciones.

Se demostró que el área de Broca (BA 45) se activaba significativamente cuando el señante nombraba objetos sin establecer relación espacial de ningún tipo, pero cuando se introducía dicha relación a través de clasificadores, la activación decrecía notablemente. En cambio, cuando el mismo sujeto realizaba la misma tarea en lengua oral se comprobaba que tanto para la designación de los objetos como para la relación entre estos mediante una preposición la activación del área de Broca era parecida. Por tanto, y según se desprende de los resultados de este experimento, en el área de Broca no se procesaría la producción de clasificadores. Ante esto cabe una pregunta: ¿cuál es el motivo de esto? Emmorey (2006) cree que se debe a que, en realidad, dichos clasificadores no constituyen una categoría lingüística como lo pueden constituir los sustantivos o las preposiciones, y por tanto se procesarían fuera del área de Broca.

Para apoyar esta hipótesis, se le pidió a los señantes que usaran preposiciones en ASL. Las preposiciones en ASL son una clase de palabras que no funcionan como las preposiciones en la lengua inglesa. De hecho, apenas se usan en ASL, y cuando se hace tienen un carácter meramente enfatizador. En este ensayo, se pudo comprobar cómo el área de Broca se activó en la producción de estos elementos marginales en ASL. Es decir, que el comportamiento de las preposiciones en ASL es parecido al de las preposiciones en la lengua oral inglesa a nivel neuronal. En este caso ambos pertenecen a la misma categoría, junto a sustantivos y verbos, y por tanto son susceptibles de ser activadas en la misma área del cerebro responsable de este proceso: el área de Broca (Emmorey, 2006). 
Se demuestra, en virtud de los resultados obtenidos en los experimentos vistos arriba, que el área de Broca no está especializada exclusivamente en la fonética de las lenguas orales. Las lesiones cerebrales en el hemisferio izquierdo en esta área producen síntomas en usuarios de la ASL parecidos a los que sufren usuarios de la lengua oral: afasia no- fluente que presupone dificultad en la configuración y déficit significativo en la coordinación de las dos manos que se puede corresponder a la realización forzosa e imprecisa en la lengua oral.

Así mismo, se comprueba que el área de Broca se activa para categorías lingüísticas tales como sustantivos, verbos o preposiciones, pero no para los clasificadores que permiten representar relaciones espaciales entre objetos, quizá porque no formen parte de las categorías gramaticales de la lengua. Se ha visto, no obstante que cuando se usan preposiciones en ASL, elementos enfatizadores, el área de Broca sî experimenta un incremento de actividad puesto que estos elementos sí se incluyen en las categorías procesadas por el área 44 de Brodmann (BA44).

\section{Evidencias Psicolingüísticas: el Idioma de Signos Nicaragüense}

Habitualmente los gestos que acompañan al habla se consideran maleducados y, por tanto, son frecuentemente, aunque todos los usamos inconsciente e inevitablemente ya que forman parte de nuestra manera natural de comunicarnos. ${ }^{7}$ Tradicionalmente, las lenguas de señas se han relacionado de una u otra manera con los gestos del habla y como consecuencia, muchos padres deciden que sus hijos no la adquieran desde pequeños optando por la lengua oral. Esta situación provoca en ocasiones problemas de cognición y de socialización puesto que tener una lengua desde edad temprana es una herramienta imprescindible para el desarrollo cognitivo y social del niño. Durante muchos años las instituciones públicas han fomentado la enseñanza de la lengua oral a niños sordos. Los resultados no han sido la mayoría de las veces los esperados por sus promotores puesto que no todos los niños sordos pueden conseguir adquirir una lengua oral. ${ }^{8}$ Como consecuencia estos niños se ven privados de una herramienta cognitiva vital para su desarrollo. Este problema se evitaría si estos niños tuvieran acceso desde pequeños a la lengua de señas, un sistema lingüístico que se adquiere de manera natural y que como otras lenguas orales el único requisito que necesita es la inmersión lingüística desde pequeños.

\footnotetext{
${ }^{7}$ De hecho, numerosos autores consideran que los gestos que acompañan al habla son parte de nuestra herencia genética y, por tanto, no pueden ser evitados en la comunicación.

${ }^{8}$ Hay muchas variables que condicionan que un niño pueda o no adquirir una lengua oral, por ejemplo, es necesario diferenciar entre distintos tipos de sordera dependiendo, fundamentalmente, a si la pérdida de la audición se ha producido después del nacimiento o no. Evidentemente, una persona que se ha quedado sorda después de haber adquirido el lenguaje oral (poslocutivo), podrá seguir comunicándose con él el resto de su vida.
} 
Sabemos que las lenguas de señas son adquiridas por los signantes utilizando las mismas herramientas cognitivas que los hablantes a través de por numerosos estudios psicolingüísticos y neurolingüísticos realizados hasta el momento (véase Petitto et al. 2004) y por el primer caso investigado del proceso de creación de una nueva lengua de señas: la Lengua de Señas Nicaragüense (LSN). La Lengua de Señas Nicaragüense surgió como un código rudimentario de señas caseras y sin estructura lingüística que en solo veinticinco años se ha convertido en un sistema lingüístico completo, en una lengua natural debido a su adquisición por parte de los niños pequeños. Esta lengua conocida en la actualidad como Idioma de Signos Nicaragüense (ISN) ha sufrido un proceso de criollización, que pasamos a reseñar seguidamente (Senghas y Kegl, 1994; Senghas et al. 1997; Kegl, 2000; Kegl, 2004; Senghas, et al., 2004).

En los años ochenta del siglo XX, el nuevo gobierno sandinista nicaragüense decidió que la educación sería una de las áreas prioritarias de la revolución para desarrollar el país. El objetivo: que todo nicaragüense fuera alfabetizado. Una de las medidas que se tomaron fue la creación de una escuela especial para sordos en la que se educara en el método oralista. ${ }^{9}$ Hasta aquella época, no había existido una lengua de señas nicaragüense sino que los sordos hacían uso de códigos de comunicación gestual caseros que usaban con sus familias.

Durante los primeros cursos, los profesores prohibían el uso de las señas en clase, pero durante el resto del tiempo los niños se comunicaban por gestos mediante un código que poco a poco se iba enriqueciendo. Judy Kegl, que trabajó durante aquel tiempo en Nicaragua, se dio cuenta de un fenómeno muy interesante: años después de que los que niños se incorporan al colegio, el sistema lingüístico fue mejorando hasta el punto de que el código basado en signos caseros y poco estructurado que surgió en un principio se fue tornando en una lengua natural, convirtiéndose en el Idioma de Signos Nicaragüense. Este fenómeno se conoce en lingüística como criollización.

La criollización ha sido constatada en situaciones de uso de códigos orales artificiales como, por ejemplo, durante la emigración que se produjo en Hawai en los años 20 del siglo XX (Pinker, 1994). Los emigrantes llegados a Hawai constituían una comunidad lingüística heretogénea que carecía de una lengua común para la comunicación. Como ocurre habitualmente en este tipo de situaciones, crearon un código artificial o pidgin para la comunicación. ${ }^{10}$ Las siguientes generaciones nacidas de esos emigrantes adquirieron dicho pidgin de manera natural, de modo que se

\footnotetext{
${ }^{9}$ Método educativo por el que los niños sordos aprenden una lengua oral.

${ }^{10} \mathrm{El}$ código inventado, o lengua pidgin, consiste en sistema de comunicación acordado en el que se utiliza un número limitado de signos de manera asistemática pero que con el contexto de la comunicación puede deducirse la intencionalidad del hablante. La lengua criolla, en cambio, es un sistema lingüístico pleno con estructura en el que pueden comunicarse multitud de pensamientos o ideas sin que se produzcan las ambigüedades que se crean en las lenguas pidgins.
} 
convirtió en su lengua nativa. Estas lenguas pasaron a llamarse lenguas criollas. La criollización, por tanto, consiste en la metamorfosis que experimenta un código artificial inventado por un grupo social para un fin comunicativo concreto debido a que los niños pequeños la adquieren como una lengua natural (Bickerton, 1984). ${ }^{11}$

En resumen, el proceso de criollización se produce gracias a la Facultad Humana del Lenguaje que genera tanto lenguas orales como lenguas de señas. Este proceso ampliamente estudiado y analizado por investigadores permite responder afirmativamente a una de las respuestas que abiertamente se ha planteado en este artículo: ¿se adquiere la lengua de señas utilizando los mismos mecanismos cognitivos que las lenguas orales? El caso del Idioma de Signos Nicaragüense nos enseña que sí.

\section{CONCLUSiOnes}

A modo de conclusión podemos decir que la lengua de señas es el reflejo de la Facultad Humana del Lenguaje y se diferencia de la oral principalmente en el canal o modalidad gestual-visual que la condiciona. Las lenguas de señas no son similares, de ninguna de las maneras, al sistema de comunicación que utilizan los primates no humanos. Además hemos comprobado cómo esa Facultad es específica del ser humano, y no puede ser adquirida por otras especies. El hecho de que los primates no humanos solo puedan usarla como un niño de dos años y medio demuestra que sus propiedades son tan complejas como la de las lenguas orales. Esta Facultad ya está implementada genéticamente. Al respecto hemos reseñado algunos trabajos realizados sobre personas que sufren de TEL. Como se señaló arriba, parece ser que los individuos afectados por esta dolencia tienen una mutación en un gen al que han denominado FOXP2.

Hemos mencionado trabajos de los que hoy por hoy son considerados padres de la moderna Neurolingüística: Wernicke y Broca. Sus descubrimientos sentaron las bases científicas para el estudio de los trastornos del lenguaje, especialmente de las afasias. Señalaron que el lenguaje se procesa y programa en el hemisferio izquierdo del cerebro (área de Broca para la producción, y área de Wernicke para la comprensión). Las lenguas de señas también se procesan en las áreas tradicionalmente asignadas para la producción y la comprensión de la lengua oral, aunque la actividad de las áreas visuales se incrementa de manera significativa en señantes. $\mathrm{Al}$ respecto, se ha visto cómo el área de Broca desempeña un papel de primer orden en la producción de la lengua de señas. El lenguaje no tiene por qué asociarse al sonido de manera exclusiva, sino también al canal visual. Es decir, el cerebro posee la suficiente plasticidad para adaptarse a dos modalidades distintas. Oralidad y gestualidad son, pues, modalidades lingüísticas igualmente naturales.

${ }^{11}$ El Bioprograma de Bickerton (1984) no es la única tesis que hoy en día explica el proceso de criollización. Véase Chaudenson (1979) para la tesis superestratista; Lefebvre (1998) para la tesis sustratista o, Mufwene (2001) y DeGraff (2003), para otras hipótesis universalistas. 
No tiene por tanto cabida el falso y dañino prejuicio de que las lenguas de señas son códigos artificiales que pueden ser aprendidos de adultos como si se tratara del código de la circulación, y que por tanto es secundaria a la lengua oral, un prejuicio que ha provocado en la comunidad lingüística sorda un sentimiento de inferioridad injustificado. Precisamente, a favor de esa naturalidad hemos aportado el caso del ISN (antes LSN), un argumento de peso que demuestra que estas lenguas cuentan con reglas recursivas, división de oraciones en sintagmas, y éstos a su vez en piezas léxicas debidamente ordenadas en jerarquías dentro del constituyente.

Sin duda alguna, la situación social de las personas sordas es sensible a estos prejuicios que han hecho que muchos padres de niños sordos no consideren siquiera la posibilidad de que sus hijos adquieran de pequeños la LSE. Las consecuencias son en ocasiones muy negativas para el desarrollo cognitivo y emocional del vástago. Incluso, los prejuicios sociales, que creíamos desterrados, todavía son moneda de uso corriente incluso en ambientes académicos. No es extraño encontrar personas formadas académicamente que desgraciadamente aún sostienen que la lengua de señas es inferior a cualquier lengua oral.

Este artículo intenta desmontar estos prejuicios. Las lenguas de señas son lenguas naturales propias del ser humano que se adquieren de manera natural y se procesan en el cerebro de la misma forma que lo hacen las orales (aunque existe una mayor actividad en el lóbulo occipital).

Porque es una perversión creer que nuestra lengua oral es superior a la de señas por el mero hecho de que hayamos nacido oyentes.

\section{REFERENCIAS BIBLIOGRÁFICAS}

BICKERTON, D. (1984), The language bioprogram hypothesis, The Behavioral and Brain Sciences 7.2, 173-221.

Bloomfield, L. (1933) Language, Chicago, Chicago University Press, edición de la Universidad de Chicago de 1984.

CAPlan, D. (1988), Biologic Foundations of Language, en Newmeyer (comp.) Linguistics: The Cambridge Survey III. Language: Psychological and Biological Aspects, Cambridge: CUP. [trad. Panorama de la Lingüística Moderna, (1992) Madrid: Visor].

Chaudenson, R. (1979), Les Créoles Francais. Paris: Nathan.

Chomsky, N. (1965), Aspects of the Theory of Syntax, Cambridge: MIT Press.

Corina D.P, McBurney S.L., Dodrill C., Hinshaw K., Brinkley J, Ojemann G. (1999), Functional roles of Broca's area and supramarginal gyrus: Evidence from cortical stimulation mapping in a deaf signer, NeuroImage, 10:570-581.

CORINA, D.P (1998), Aphasia in users of signes language, en P. Coppens et. al. (eds), Aphasia in atypical populations, Mahwah, NJ: Lawrence Erlbaum Associates págs. 261-310. 
DeGraff, M. (2003), Against Creole Exceptionalism, Language 79.2:391-410.

Emmorey, K. (2002), Language, Cognition, and the Brain. Insights from Sign Language Research, Mahwah, NJ and London: Lawrence Erlbaum.

EMmorey, K. (2006), The role of Broca's area in sign language, en Y. Grodzinsky et. al (eds). Broca's Region, Oxford: Oxford University Press.

EMMOREY, K. y M. Herzig (2003), Categorial versus gradient properties of classifier constructions in ASL, en K. Emmorey (ed.), perspective on classifier construction in signed language, Mahwah, NJ: Lawrence Erlbaum Associates, págs. 222-246.

GARDNER, R.A. y Gardner, B.T. (1969), Teaching sign language to a chimpanzee. Science, 165: 664-72.

GESCHWIND, N. (1965), Disconnection syndromes in animal and man, Brain, 88: 23794.

GRODZINSKY, Y. (2000) The neurology of syntax: language use without Broca's area. Behavioural and Brain Sciences, 23:1-73.

GroDZINSKY, Y. y L. Finkel (1998), The Neurology of empty categories: aphasics' failure to detect ungrammaticality, Journal of Cognitive Neuroscience, 10:2:281-292.

HAuser, M., N. Chomsky y T. Fitch (2002), The faculty of language: what is it, who has it and how did it evolve?, Science 298:1569-1579.

HickOK, G., Love-Geffen, T., y E.S. Klima (2002), Role of the left hemisphere in sign language comprehension, Brain and Language, 82:67-178.

HICKOK, G., U. Bellugi y E.S. Klima (2002), Sign language in the brain, Scientific American, 12: 45-53.

Kegl, J. (2000), Is it soup yet? or, When is it Language?, en Proceedings of the Child Language Seminar 1999. City University, London.

KEGL, J. (2004), Language Emergence in a Language-Ready Brain: Acquisition Issues, en Jenkins, Lyle, (ed), Biolinguistics and the Evolution of Language. Amsterdam:John Benjamins.

KLIMA, E.S. y U. Bellugi (1979), The signs of language, Cambridge: Harvard University Press.

LAI, C. S. L., Fisher, S. E., Hurst, J. A., Vargha-Khadem, F., and Monaco, A. P. (2001), A forkhead-domain gene is mutated in a severe speech and language disorder, Nature, 413:519--523.

LEFEBVRE, C. (1998), Creole genesis and the acquisition of grammar: Haitian creole, Cambridge: Cambridge University Press.

LIEBERMAN, P. (1984) The biology and evolution of language, Cambridge: Harvard University Press.

LIEBERMAN, P. (1991), Uniquely human. The evolution of speech, thought, and selfless behaviour, Cambridge: Harvard University Press. 
LiEBERMAn, P. (2006), Toward an evolutionary biology of language, Harvard University Press.

LIEBERMAN, P. (2007), The evolution of human speech, Current Anthropology 48,(1):3966

Mufwene, S. (2001a), The Ecology of Language Evolution,. Cambridge: Cambridge University Press.

PetitTo, L.A., R. J. Zatorre, K.Gauna, E. J. Nikelski, D. Dostie y A.C. Evans (2000), Speech-like Cerebral Activity in Profoundly deaf people while processing signed languages: implications for the neural basis of all human language, Proceedings of the National Academy of Sciences, 97 (25):13961-6.

PetTito, L.A. (2000), On the Biological Foundations of Human Language en K. Emmory y H. Lane (eds.), The signs of language revisted: An anthology in honor of Ursula Bellugi and Edward Klima. N.J.: Lawrence Erlbaum Assoc. Inc.

PetitTo, L. A., S. Holowka, L.E. Sergio, B. Levy y D.J. Ostry. (2004). Baby hands that move to the rhythm of language: Hearing babies acquiring sign language babble silently on the hands. Cognition, 93: 43-73.

PinKeR, S. (1994), The language instinct, New York: William Morrow and Company.

POIZNER, H., E. Klima y U. Bellugi (1987), What the hands reveal about the brain? Cambridge: MIT PRESS.

REYes Tejedor, M. y V. Camacho Taboada (2007), Patologias del Lenguaje: Lingüistica, afasias y agramatismos, Madrid: Editorial Universitaria Ramón Areces.

SANDLER, W. y D. Lillo-Martin (2001), Natural sign languages, en M. Aronoff y J. Rees-Miller (eds.), The Handbook of Linguistics, págs. 533-562.

SANDlER, W. y D. Lillo-Martin (2006), Sign Language and Linguistic Universals. Cambridge: Cambridge University Press.

Savage-Rumbaugh, S. (2000), Linguistic, Cultural and Cognitive Capacities of Bonobos (Pan paniscus), Culture \& Psychology, Vol. 6(2): 131-153.

SengHAS, A., S. Kita, and A. Özyürek (2004), Children creating core properties of language: evidence from an emerging sign language in Nicaragua, Science, 305: 5691, 1779-1782.

SENGHAS, A., M. Coppola, E.L. Newport y T. Supalla (1997). Argument structure in Nicaraguan Sign Language: The emergence of grammatical devices, en E. Hughes, M. Hughes y A. Greenhill (eds.), Proceedings of the 21st Annual Boston University Conference on Language Development: Vol.2. Somerville, MA: Cascadilla Press.

SENGHAS, R. y J. Kegl. (1994), Social Considerations in the Emergence of Idioma de Signos Nicaragüense (Nicaraguan Sign Language). Signpost, 7(1):40-46.

STOKOE, W.C., (1960/1978), Sign Language Structure. Silver Spring: Linstok Press. 
Terrace, H. S., Pettito, L. A., Sanders, R. J., y Bever, T. G. (1979), Can an ape create a sentence?, Science, 206: 891-902.

WILKINS, W.K., Wakefield, J. (1995), "Brain evolution and neurolinguistic preconditions", Behavioral and Brain Sciences, 18: 61-182.

Mariano Reyes Tejedor

mreytej@upo.es

Depto. de Filología y Traducción

Universidad Pablo de Olavide

Carretera de Utrera km.1

41013 Sevilla (España)
Fecha de recepción: 1/03/2007

Fecha de aceptación:10/06/2007 